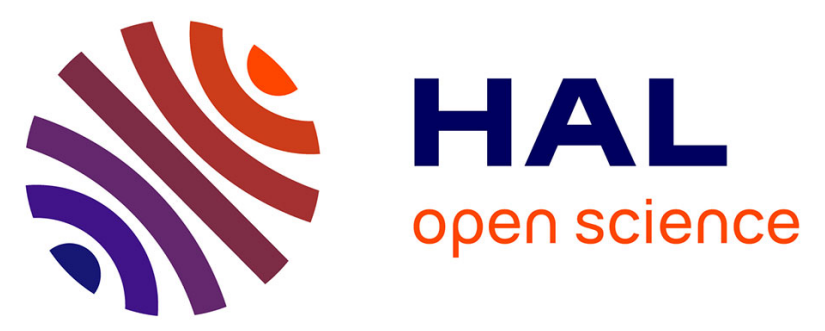

\title{
Action de la concentration de la solution nutritive sur quelques caractéristiques physiologiques et technologiques chez Vitis Vinifera L. cv. Cabernet-Sauvignon. III. - Régime hydrique, photosynthèse brute et corrélations intra-plante
}

Alain Carbonneau

\section{To cite this version:}

Alain Carbonneau. Action de la concentration de la solution nutritive sur quelques caractéristiques physiologiques et technologiques chez Vitis Vinifera L. cv. Cabernet-Sauvignon. III. - Régime hydrique, photosynthèse brute et corrélations intra-plante. Agronomie, 1984, 4 (6), pp.535-541. hal00884669

\section{HAL Id: hal-00884669 \\ https://hal.science/hal-00884669}

Submitted on 1 Jan 1984

HAL is a multi-disciplinary open access archive for the deposit and dissemination of scientific research documents, whether they are published or not. The documents may come from teaching and research institutions in France or abroad, or from public or private research centers.
L'archive ouverte pluridisciplinaire HAL, est destinée au dépôt et à la diffusion de documents scientifiques de niveau recherche, publiés ou non, émanant des établissements d'enseignement et de recherche français ou étrangers, des laboratoires publics ou privés. 


\title{
Action de la concentration de la solution nutritive sur quelques caractéristiques physiologiques et technologi- ques chez Vitis Vinifera L. cv. Cabernet-Sauvignon. III. - Régime hydrique, photosynthèse brute et corréla- tions intra-plante.
}

\author{
Alain CARBONNEAU
}

I.N.R.A., Station de Recherches de Viticulture, Centre de Recherches de Bordeaux, F 33140 Pont-de-la-Maye

Mots clés additionnels : Vigne, résistance stomatique, potentiel hydrique.

Effect of nutrient solution concentration on some physiological and technological characteristics in Vitis vinifera L. cv. Cabernet-Sauvignon. III. - Water flow, gross photosynthesis, intra-plant correlations.

The variation of grape sugar content in relation to nutrient solution concentration could be explained by results
on gross photosynthesis. The particular effect of high nutrient solution concentration on gross photosynthesis
seemed to involve first a higher degree of root branching accompanied by lower occupation of the available
volume, then a decrease in water flow through the whole plant, and finally a reduction in $\mathrm{CO}_{2}$ assimilation
per unit leaf area.

Additional key words : Grapevine, stomatal resistance, water potential.

\section{INTRODUCTION}

Parmi les facteurs écologiques agissant sur la production et la maturation du raisin, il est intéressant de situer le rôle des éléments minéraux considérés sous l'angle de leur concentration dans l'environnement racinaire. En outre, il apparaît primordial de mesurer l'influence de ce facteur sur certains éléments de base de l'activité foliaire : la photosynthèse brute, la résistance stomatique et le potentiel de sève. Enfin, l'étude de tels phénomènes dans un contexte « plante entière » impose, pour interpréter les résultats, de mesurer ou de maitriser les corrélations intra-plante au niveau des principales fonctions physiologiques et des connexions entre organes.
Une telle approche méthodologique a été utilisée par CARBONNEAU et al. (1978) et par CARBONNEAU $(1978,1980 a)$ pour mettre en évidence des effets assez directs du microclimat au sein de la végétation sur la production et la maturation du raisin, effets liés pour l'essentiel au métabolisme foliaire (ex. : photosynthèse brute) et beaucoup moins aux relations entre organes (ex. : répartition des sucres). A cette occasion, il a été noté quelques différences de teneur en éléments minéraux des limbes et des pétioles en fonction du microclimat, mais sans rapport avec les paramètres précédents.

D'autres études (CARBONNEAU et al., 1982 ; CARBONNEAU et al., 1983) ont montré que certaines fluctuations de la nature du sol autour d'un même type 
général de sol de " graves " entraînent des variations significatives de la teneur en eau et en éléments minéraux majeurs des feuilles. Mais, dans ce cas, les écarts de production et de maturation s'expliquent essentiellement par les variations induites du microclimat au sein de la végétation; de même, les différences de photosynthèse brute sont surtout liées à ces mêmes variations microclimatiques ainsi qu'à l'abaissement des résistances internes rencontrées par le $\mathrm{CO}_{2}$ en fonction de l'augmentation du niveau de croissance des feuilles. Les éléments minéraux n'ont donc pas joué ici un rôle inducteur prédominant de variations photosynthétiques.

De ce fait, de tels effets doivent être recherchés dans des essais où l'alimentation minérale est fournie à la plante, d'une part, avec des niveaux très différents et, d'autre part, de façon contrôlée en maîtrisant les autres facteurs du milieu. Cette démarche a été suivie par ALLEWELDT (1971) ; elle a montré que la photosynthèse nette présente un maximum d'intensité, très peu précis du reste, pour certaines teneurs de la solution nutritive en $\mathrm{N}, \mathrm{P}, \mathrm{K}$, pris de façon isolée ou combinée. Toutefois, cette expérience a révélé principalement des effets soit de toxicité, soit de carence et a, par conséquent, apporté peu de résultats pour la gamme de concentration d'éléments minéraux compatible avec une croissance normale de la plante.

En ce qui concerne plus spécifiquement l'azote, CHAMPIGNY (1960) a mis en évidence une chute de la photosynthèse par unité de surface foliaire consécutive à l'augmentation de l'absorption des nitrates et à leur réduction dans les feuilles. Ce mécanisme a été invoqué par CHAMPAGNOL (1971) pour expliquer la baisse de photosynthèse brute et de teneur en sucres des baies en fonction de l'augmentation de la dose d'azote dans des essais de fertilisation. Toutefois, l'influence du microclimat de la végétation (qui est fortement modifiable par l'effet stimulant de l'azote à l'égard de la surface foliaire) sur la photosynthèse n'a pas été pris en compte, ce qui ne permet pas de mesurer l'importance du mécanisme relatif à la réduction des nitrates.

De même, dans beaucoup d'essais de fertilisation, en raison des multiples influences des minéraux par exemple sur la croissance des tiges et des baies, sur la fécondation, ou sur la morphologie racinaire - aucune donnée ne permet à l'heure actuelle de démontrer chez la vigne un effet de la teneur en éléments minéraux sur la photosynthèse brute en particulier. L'expérience décrite ci-après tente de pallier cette insuffisance.

\section{MATÉRIEL ET MÉTHODES}

Le dispositif expérimental est constitué de vases de végétation plantés en "Cabernet-Sauvignon 》 greffé sur plusieurs porte-greffes et alimentés avec 2 régimes saturants d'irrigation localisée fertilisante (S et $2 S$ ) différant par le niveau de concentration des éléments minéraux (POUGET, 1984).

En 1981, des observations systématiques sur chacune des plantes de l'essai ont permis de visualiser certains phénomènes décrits ci-après et de choisir 4 plantes représentatives de la moyenne de vigueur par niveau de concentration; ces individus correspondent au seul porte-greffe " Fercal».

En juillet 1982, les méthodes particulières suivantes ont été utilisées :

- croissance et surface foliaire (CARBONNEAU, $1976 a$ et $1976 b)$;

- potentiel de sève foliaire (chambre à pression du type SCHOLANDER) mesuré en milieu de journée par ciel clair ;

- résistance stomatique et photosynthèse brute avec un poromètre à diffusion modèle "Cayuga " (CARBONnEAU, 1983), mesurées en milieu de journée par ciel clair ;

- éclairement des feuilles (quantum-mètre "Lambda ») mesuré en milieu de journée par ciel clair.

Les autres notations ont été effectuées sur les 8 plantes de chaque traitement. Les mesures de ces divers paramètres sont complétées par des observations du fonctionnement de la "plante entière » au sein duquel il est nécessaire d'estimer le niveau des principales fonctions (CARBONNEAU, 1980a). Sans prétendre atteindre la mesure de toutes les corrélations intra-plante, il a été noté au total :

- le poids des racines, du tronc et des bras (estimant une fonction d'investissement à long terme de la partie pérenne de la plante),

- le nombre et le poids moyen des grappes (estimant la fertilité ou une fonction d'investissement à court terme),

- la photosynthèse brute et la teneur des limbes et pétioles en éléments minéraux majeurs (estimant des fonctions de production de la feuille),

- la résistance stomatique et le potentiel de sève foliaire (estimant une fonction de production des feuilles en équilibre très étroit avec l'activité racinaire),

- la croissance des tiges et des feuilles, le poids des raisins, la répartition de l'appareil racinaire dans l'espace du vase de végétation (estimant une fonction de consommation ainsi que le développement des interfaces «feuille-air » et « racine-sol »),

- la teneur en sucres solubles et insolubles totaux des sarments aoûtés en pourcentage de la matière sèche (estimant une fonction d'épargne dans la partie végétative),

- la teneur des raisins à maturité en sucres réducteurs totaux et en acidité totale (estimant une fonction d'épargne dans la partie productrice).

\section{RÉSULTATS}

Le schéma du fonctionnement de la plante entière peut se résumer ainsi, relativement aux 2 types de solution $\mathrm{S}$ et $2 \mathrm{~S}$ :

- la fonction d'investissement à long terme (poids des racines, du tronc et des bras) atteint les mêmes niveaux pour les 2 types de solution (tabl. 1) ;

- la fonction d'investissement à court terme (nombre et poids moyen des grappes, d'après POUGET, 1984) également ; 
TABLEAU 1

Résultats physiologiques du cycle végétatif de 1982 concernant les 2 solutions nutritives $S$ et $2 S$ et le Cabernet-Sauvignon/Fercal Physiologicat results for the 1982 growing season on the effect of the 2 nutrient solutions $S$ and $2 S$ on Cabernet-Sauvignon/Fercal

\begin{tabular}{|c|c|c|c|c|c|c|c|c|c|c|}
\hline \multirow[b]{2}{*}{ Paramètres } & \multicolumn{3}{|c|}{ Solution S } & \multicolumn{3}{|c|}{ Solution $2 S$} & \multirow[b]{2}{*}{$\begin{array}{l}\text { Nombre } \\
\text { de } \\
\text { répéti- } \\
\text { tions }\end{array}$} & \multirow[b]{2}{*}{$\begin{array}{l}\text { Signi- } \\
\text { fica- } \\
\text { tion }\end{array}$} & \multirow[b]{2}{*}{$\begin{array}{l}\text { Période } \\
\text { d'obser- } \\
\text { vation }\end{array}$} & \multirow[b]{2}{*}{ Remarques } \\
\hline & $\begin{array}{l}\text { limite } \\
\text { infé- } \\
\text { rieure } \\
\text { à } 5 \%\end{array}$ & $\begin{array}{l}\text { moyen- } \\
\text { ne }\end{array}$ & $\begin{array}{l}\text { limite } \\
\text { supé- } \\
\text { rieure } \\
\text { à } 5 \%\end{array}$ & $\begin{array}{l}\text { limite } \\
\text { infé- } \\
\text { rieure } \\
\text { à } 5 \%\end{array}$ & $\begin{array}{l}\text { moyen- } \\
\text { ne }\end{array}$ & $\begin{array}{l}\text { limite } \\
\text { supé- } \\
\text { rieure } \\
\text { à } 5 \%\end{array}$ & & & & \\
\hline Poids du tronc et des bras (g) & 436 & 515 & 594 & 500 & 561 & 622 & 8 & $\begin{array}{l}\text { NS } \\
\text { (Stu- } \\
\text { dent) }\end{array}$ & $9 / 3 / 83$ & $\begin{array}{l}\text { Plants greffés sur } 7542 \text {, } \\
\text { porte-greffe induisant des } \\
\text { réponses voisines du Fer- } \\
\text { cal }\end{array}$ \\
\hline $\begin{array}{l}\text { Poids frais total des racines } \\
\text { d'un plant }(\mathrm{g})\end{array}$ & 308 & 496 & 684 & 296 & 438 & 580 & 8 & $\begin{array}{l}\text { NS } \\
\text { (Stu- } \\
\text { dent) }\end{array}$ & $9 / 3 / 83$ & Idem \\
\hline $\begin{array}{l}\text { Rapport du poids frais des } \\
\text { racines ramifiées entre la } \\
\text { moitié inférieure et la moi- } \\
\text { tié supérieure du vase de } \\
\text { végétation }\end{array}$ & $/$ & $\mathbf{1 , 5 2}$ & l & / & 0,92 & l & 8 & $\begin{array}{c}\text { S à } 5 \% \\
\text { (Kruskal } \\
\text { Wallis) }\end{array}$ & $30 / 3 / 83$ & $\begin{array}{l}\text { Plants greffés sur Rupes- } \\
\text { tris du Lot, porte-greffe } \\
\text { à morphologie racinaire } \\
\text { voisine du Fercal }\end{array}$ \\
\hline $\begin{array}{l}\text { Eclairement des feuilles } \\
\left(\mu \mathrm{E} \mathrm{m}^{-2} \cdot \mathrm{s}^{-1}\right)\end{array}$ & / & 1800 & / & 1 & 1800 & / & 80 & $\begin{array}{l}\text { NS } \\
\text { (Stu- } \\
\text { dent) }\end{array}$ & $\begin{array}{l}19-27 / \\
07 / 82\end{array}$ & $\begin{array}{l}\text { Feuilles adultes, saines, } \\
\text { bien exposées en milieu } \\
\text { de journée, sur sarments } \\
\text { situés au milieu du bois } \\
\text { de taille }\end{array}$ \\
\hline $\begin{array}{l}\text { Résistance stomatique } \\
\left(\mathrm{s} . \mathrm{cm}^{-1}\right)\end{array}$ & 2,2 & 2,4 & 2,6 & 3,1 & 3,5 & 3,9 & 80 & $\begin{array}{l}\text { S } 5 \% \\
\text { (Stu- } \\
\text { dent) }\end{array}$ & $\begin{array}{l}19-27 / \\
07 / 82\end{array}$ & Idem \\
\hline $\begin{array}{l}\text { Potentiel de sève foliaire } \\
\left(-\mathrm{kg} \cdot \mathrm{cm}^{-2}\right)\end{array}$ & $-11,6$ & $-12,3$ & $-13,0$ & $-12,1$ & $-13,2$ & $-14,1$ & 40 & $\begin{array}{l}\text { NS } \\
\text { (Stu- } \\
\text { dent) }\end{array}$ & $\begin{array}{l}19-27 / \\
07 / 82\end{array}$ & Idem \\
\hline $\begin{array}{l}\text { Photosynthèse brute } \\
\left(\text { ng } \mathrm{CO}_{2} \mathrm{~cm}^{-2} \cdot \mathrm{s}^{-1} \text { ) }\right.\end{array}$ & 40,8 & 44,7 & 48,6 & 35,6 & 38,3 & 40,9 & 40 & $\begin{array}{c}\text { S } 5 \% \\
\text { (limite) } \\
\text { (Stu- } \\
\text { dent) }\end{array}$ & $\begin{array}{l}19-27 / \\
07 / 82\end{array}$ & Idem \\
\hline Longueur du sarment $(\mathrm{cm})$ & 180,1 & 213,5 & 246,9 & 199,5 & 221,8 & 244,1 & 20 & $\begin{array}{l}\text { NS } \\
\text { (Stu- } \\
\text { dent) }\end{array}$ & $28 / 7 / 82$ & $\begin{array}{l}\text { Sarments situés au milieu } \\
\text { du bois de taille }\end{array}$ \\
\hline $\begin{array}{l}\text { Nombre de feuilles du sar- } \\
\text { ment }\end{array}$ & 29,9 & 32,5 & 35,1 & 30,3 & 32,5 & 34,7 & 20 & $\begin{array}{l}\text { NS } \\
\text { (Stu- } \\
\text { dent) }\end{array}$ & $28 / 7 / 82$ & Idem \\
\hline $\begin{array}{l}\text { Surface moyenne d'une } \\
\text { feuille sur le sarment }\left(\mathrm{cm}^{2}\right)\end{array}$ & 110,7 & 116,5 & 122,3 & 101,2 & 107,3 & 113,4 & 20 & $\begin{array}{l}\text { NS } \\
\text { (Stu- } \\
\text { dent) }\end{array}$ & $28 / 7 / 8$ & Idem \\
\hline $\begin{array}{l}\text { Surface foliaire du sarment } \\
\left(\mathrm{dm}^{2}\right)\end{array}$ & 33,7 & 38,0 & 42,3 & 31,4 & 35,0 & 38,6 & 20 & $\begin{array}{l}\text { NS } \\
\text { (Stu- } \\
\text { dent) }\end{array}$ & $28 / 7 / 82$ & Idem \\
\hline $\begin{array}{l}\text { Sucres insolubles totaux des } \\
8^{\text {e }} \text { et } 9^{\mathrm{e}} \text { mérithalles des bois } \\
\text { de taille }(\% \mathrm{MS})\end{array}$ & 22,4 & 23,1 & 23,8 & 21,9 & 22,4 & 22,9 & 8 & $\begin{array}{l}\text { NS } \\
\text { (Stu- } \\
\text { dent) }\end{array}$ & $01 / 83$ & $\begin{array}{l}\text { Prélèvement sur l'ensem- } \\
\text { ble des bois de taille }\end{array}$ \\
\hline $\begin{array}{l}\text { Sucres solubles totaux des } \\
8^{\mathrm{e}} \text { et } 9 \text { mérithalles des bois } \\
\text { de taille }(\% \mathrm{MS})\end{array}$ & 5,8 & 6,5 & 7,2 & 4,3 & 5,8 & 6,3 & 8 & $\begin{array}{l}\text { NS } \\
\text { (Stu- } \\
\text { dent) }\end{array}$ & $01 / 83$ & Idem \\
\hline
\end{tabular}

- les fonctions de production des feuilles manifestent des écarts :

- significatifs pour la résistance stomatique qui est inférieure de 30 p. 100 pour S par rapport à $2 \mathrm{~S}$ (tabl. 1),

- à la limite de la signification pour la photosynthèse brute qui est supérieure de 17 p. 100 pour
S par rapport à $2 \mathrm{~S}$ (tabl. 1),

- non significatifs pour le potentiel de sève foliaire qui est cependant légèrement supérieur en valeur négative pour $\mathrm{S}$ par rapport à $2 \mathrm{~S}$ (tabl. 1),

- significatifs pour la teneur des limbes en certains éléments minéraux (azote total notamment), d'après DELAS \& POUGET (1984) ; 
- la fonction de consommation ne fait ressortir aucune différence significative entre $S$ et $2 S$ pour les poids de raisins, de bois (POUGET, 1984) et de racines, ni pour la croissance des tiges et des feuilles (tabl. 1).

Par contre, la morphologie globale du système racinaire est très différente entre $S$ et $2 S$ (tabl. 1 et fig. 1), $S$ induisant une bien meilleure colonisation $\mathrm{du}$ vase de végétation dans la partie basse, donc loin des goutteurs, et $2 S$ provoquant une plus forte ramification $\mathrm{du}$ chevelu radicellaire mais superficiellement dans la partie supérieure du vase.

Il est à noter, dans le tableau 1, que le rapport du poids frais des racines entre la moitié inférieure et la moitié supérieure du vase de végétation est un assez médiocre estimateur des surfaces d'échange racinaire. Toutefois, ce paramètre indique significativement la même tendance qui est nettement perceptible dans la figure 1 .

La colonisation souterraine est donc plus développée dans le cas de $\mathrm{S}$, alors que la colonisation aérienne est rigoureusement identique entre $S$ et $2 S$ sur le plan de la surface et de l'éclairement des feuilles (tabl. 1).

- la fonction d'épargne est assez analogue entre $\mathrm{S}$ et $2 \mathrm{~S}$ s'agissant des réserves en sucres solubles et insolubles totaux dans les bois (tabl, 1). Par contre, la teneur en sucres des raisins à maturité est nettement plus élevée - de 15 p. 100 en moyenne - dans la solution $S$ par rapport à $2 S$ (PouGET, 1984). Cette augmentation importante est donc du même ordre que celle relative à la photosynthèse brute.

\section{DISCUSSION}

Au vu de l'ensemble de ces résultats, il apparaît que l'écart de teneur en sucres dans les raisins entre $S$ et $2 \mathrm{~S}$ est difficilement imputable à une différence de distribution des sucres entre les divers organes de la plante. En effet, la teneur en sucres des bois ainsi que les poids des organes annuels et pérennes sont très voisins entre $S$ et $2 S$.

L'écart de teneur en sucres des raisins doit donc être mis au compte d'une différence initiale de photosynthèse, ce qui a été vérifié de façon ponctuelle à la fin de la croissance de 1982, mais aussi à des stades antérieurs en 1981.

De ce fait, il est logique de penser que la meilleure photosynthèse observée dans le cas de $\mathrm{S}$, d'une part, assure une meilleure alimentation en sucres du raisin au cours de la maturation et, d'autre part, permet pendant la phase de croissance l'établissement d'une surface foliaire et d'une quantité de fruits analogues.

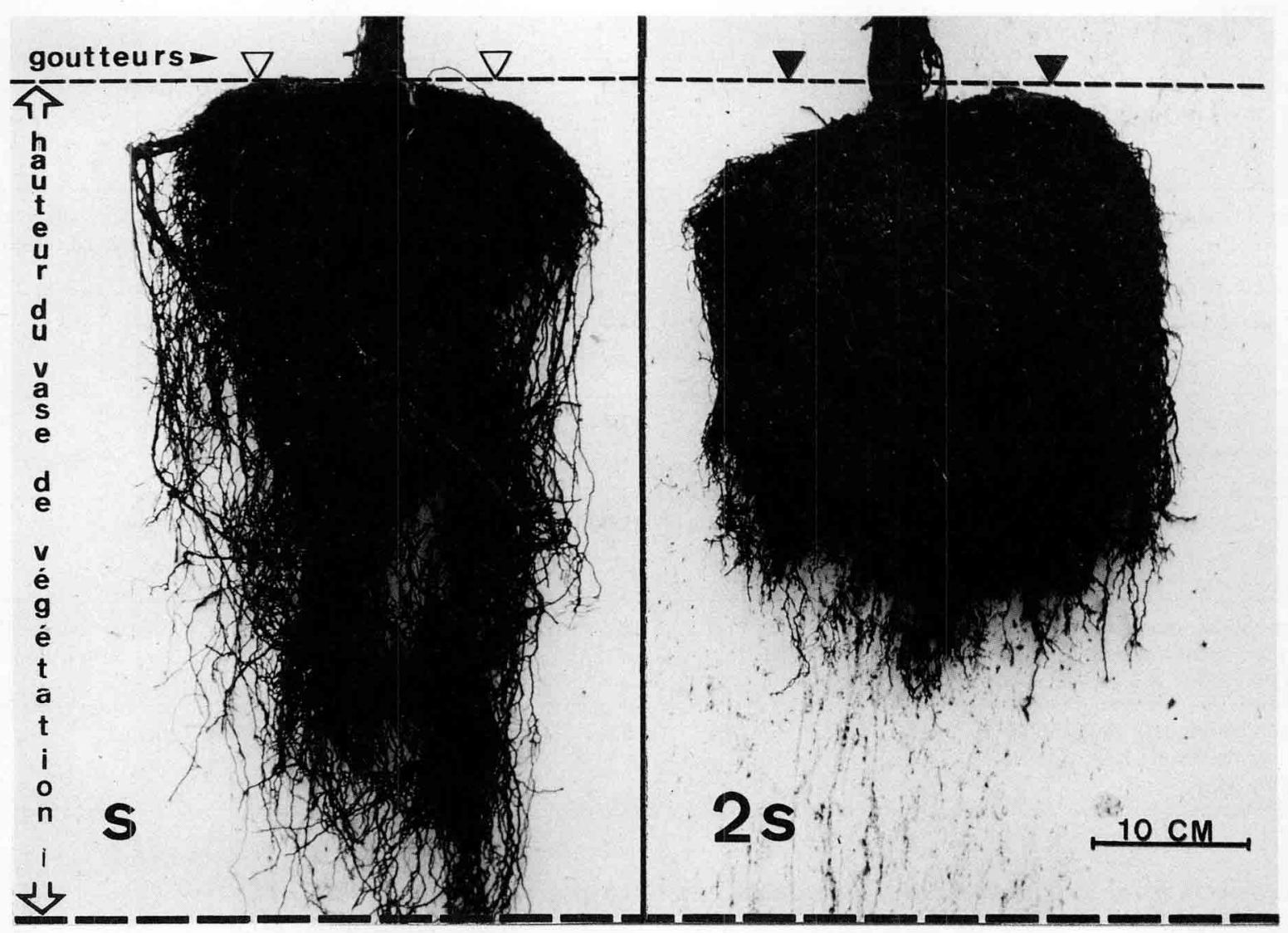

Vue de la morphologie racinaire globale pour les solutions nutritives $S$ (à gauche) et $2 S$ (à droite). Pour $S$, les racines colonisent toute la hauteur du vase de végétation; pour $2 S$, environ la moitié mais avec une densité radicellaire plus élevée.
Overall root morphology for nutrient solution $S$ (left) and $2 S$ (right). For $S$, the roots occupied the whole height of the growth container ; for $2 S$, approximatively half but with a higher rootlet density. 
Ce dernier point pourrait résulter, dans le cas de $\mathrm{S}$ et au niveau des phénomènes de croissance en général, d'une compensation d'une moindre alimentation minérale (azotée notamment) par une meilleure nutrition carbonée.

Ce phénomène de compensation n'est d'ailleurs pas très net pendant les 2 premiers cycles végétatifs (POUGET, 1984) : Ceci montre bien que l'on ne se trouve pas, dans le cas de $2 S$, en présence d'un phénomène d'excès d'alimentation minérale relativement nuisible à la croissance, mais plutôt que la situation $\mathrm{S}$ est endeçà de l'optimum de croissance vis-à-vis de l'alimentation minérale avant que l'effet de compensation par une meilleure nutrition carbonée ait pu suffisamment s'exprimer.

L'explication de la différence de photosynthèse elle-même fait appel à priori à 3 approches différentes concernant :

- le milieu racinaire ;

- le métabolisme foliaire ;

- le régime hydrique de la plante entière.

\section{A. Le milieu racinaire}

Bien que le milieu racinaire soit composé dans les 2 cas de sable grossier, lessivé entre chaque cycle végétatif, et que la seule différence contrôlée soit la concentration initiale de la solution nutritive, il n'est pas exclu que d'autres différences y existent.

En particulier, la densité très élevée du chevelu radicellaire dans le cas de $2 S$ peut accroître le taux de matière organique résultant de la décomposition des racines mortes, ce fait modifiant les capacités de rétention de l'eau au voisinage des racines. Toutefois, un tel effet paraît véritablement sans commune mesure avec les écarts de transpiration enregistrés.

De même, la densité très élevée de radicelles dans le cas de $2 \mathrm{~S}$ peut créer, par simple effet de capillarité, un assèchement dans le bas du vase de végétation limitant ainsi la colonisation de l'espace offert. Toutefois, une telle influence peut être négligée car la solution nutritive était librement coulante, sauf cependant en fin de croissance pendant quelques journées estivales à forte E.T.P.

Enfin, en fonction de l'absorption différentielle de l'eau et des minéraux par les racines, un gradient de concentration existe dans le vase de végétation et peut engendrer des effets osmotiques à certains niveaux. Cependant, un tel gradient ne doit pas être très accentué et les effets osmotiques pas très nets, car la solution nutritive initiale $2 \mathrm{~S}$ possède une pression osmotique très basse. En tout cas, l'arrêt de développement racinaire vers le milieu du vase de végétation ne peut pas être imputé à un gradient de concentration dans le vase, puisqu'au-dessus de cette limite la prolifération du chevelu est extrême et ne présente pas elle-même de gradient (fig. 1) ; en effet, les phénomènes osmotiques sont généralement très graduels.

\section{B. Le métabolisme foliaire}

Le mécanisme physiologique qu'il convient de retenir en priorité est sans doute la compétition pour le $\mathrm{NADPH}_{2}$ entre l'assimilation du $\mathrm{CO}_{2}$ et la réduction du $\mathrm{NO}_{3}$ - dans la feuille. Cette hypothèse est fondée sur les expériences de CHAMPIGNY (1960) qui a noté une diminution de photosynthèse de $25 \mathrm{p}$. $100 \mathrm{chez}$ une feuille qui réduit intensément les nitrates.

L'analogie est donc grande avec les résultats de l'essai, relatifs pour $2 \mathrm{~S}$ à la fois à la plus faible photosynthèse et à la plus forte teneur en azote du limbe. Toutefois, l'effet primaire au niveau de l'assimilation du $\mathrm{CO}_{2}$ concernerait ici les résistances internes avant d'affecter la résistance stomatique. De ce fait, les variations en pourcentage de la photosynthèse entre $S$ et $2 \mathrm{~S}$ devraient logiquement être plus grandes que celles de la résistance stomatique. Or l'inverse est observé.

Mais le point le plus important qui fait écarter cette hypothèse comme étant la seule et principale cause des faits observés est qu'elle apparaît incapable d'expliquer les différences de morphologie racinaire. Il est donc logique de supposer que l'effet premier et principal de la concentration de la solution nutritive ne se situe pas au niveau foliaire mais plutôt au niveau racinaire, comme le montre le modèle suivant.

\section{Le régime hydrique de la plante entière}

La base du raisonnement est de faire intervenir un effet direct de la concentration de la solution nutritive initiale sur la morphologie racinaire et l'absorption hydrique.

Le fait même d'une plus forte concentration en éléments minéraux pour $2 \mathrm{~S}$ entraînerait une ramification plus dense du chevelu radicellaire, en privilégiant la croissance latérale, cette ramification étant engendrée par des mécanismes d'ajustement osmotique ou d'ordre métabolique (points peu précisés actuellement au niveau racinaire, voire à celui des mycorhizes). Il s'agirait ici en tout cas d'un effet très localisé et immédiat de la concentration de la solution nutritive sur la morphologie racinaire et l'absorption des éléments minéraux ; ceci est très apparent dans le cas d'une fertirrigation en goutte à goutte et assez largement démontré dans d'autres conditions : par exemple chez l'orge (DREW \& SAKER, 1975) ou chez le maïs (DUNCAN \& OHLROGGE, 1958).

Outre son effet direct sur la densité même du chevelu racinaire, cette stimulation de la ramification des racines polariserait plutôt latéralement dans le cas de $2 \mathrm{~S}$ la colonisation de l'espace du vase de végétation par le système racinaire tout entier. De ce fait, une limitation de l'expansion de ce dernier se produirait assez vite par sa rencontre avec les parois verticales du vase. Ceci créerait un système racinaire très dense mais relativement superficiel, ces deux phénomènes aboutissant à l'égalité de biomasse racinaire observée entre $S$ et $2 S$.

De plus, il faut supposer, d'une part, que l'effet de la concentration de la solution nutritive sur la croissance latérale des racines se maintient ou ne change pas de nature malgré l'apparition de cette limitation physique et, d'autre part, qu'un certain équilibre s'établit entre la croissance racinaire et la croissance des sarments puisque tous les phénomènes de croissance analysés plus loin sont comparables entre $\mathrm{S}$ et $2 \mathrm{~S}$. La constance de ce rapport a été souvent observée dans des conditions d'alimentation en eau données 
(RICHARDS \& ROWE, 1977 $a, b$; CARBONNEAU, $1980 b$ ).

$\mathrm{Au}$ total, dans le cas de $\mathrm{S}$, la plante accroîtrait l'étendue de son contact avec le milieu du vase de végétation, en particulier en profondeur où existe toujours une certaine réserve hydrique en raison de la position latérale des orifices de drainage. De ce fait, l'alimentation hydrique de la plante serait meilleure, ce qui expliquerait, en premier lieu, une moindre résistance stomatique, ainsi peut-être qu'un potentiel de sève légèrement moins bas en valeur négative pour des conditions de transpiration supérieures. Il faut supposer que cette meilleure alimentation hydrique n'est pas suivie d'une compensation au niveau de l'alimentation minérale par rapport à la solution $2 \mathrm{~S}$.

En ce qui concerne l'activité foliaire, cette absorption différentielle d'eau et de minéraux ne serait pas sans conséquence. Pour $\mathrm{S}$, la meilleure transpiration créerait les conditions d'une photosynthèse par unité de surface foliaire supérieure. Pendant la phase de croissance, qui est évidemment sous la dépendance conjointe des métabolismes glucidique et azoté, le métabolisme glucidique plus intense pour $\mathrm{S}$ pallierait le moindre niveau du métabolisme azoté pour aboutir progressivement à l'installation d'une quantité globale de biomasse analogue au cas de $2 \mathrm{~S}$ (avec vraisemblablement des différences dans la composition biochimique de cette biomasse). Pendant la phase de maturation, le métabolisme glucidique est le plus important pour la plante et, à ce stade là, les plantes soumises à la solution $\mathrm{S}$ accumuleraient plus de sucres dans les raisins qui constituent le centre d'appel le plus efficace, ceci en raison à la fois d'une surface foliaire identique et d'une photosynthèse par unité de surface foliaire supérieure.

Ce modèle explicatif de la plante entière paraît assez bien rendre compte de tous les faits observés. Il ouvre la voie, d'une part, à des études fondamentales visant à vérifier les hypothèses avancées ici et, d'autre part, à mieux définir les conditions de culture en vase de végétation qui soient aptes notamment à assurer la colonisation totale de l'espace offert quel que soit le niveau de la concentration de la solution nutritive.

\section{CONCLUSION}

En dépit des particularités du dispositif expérimental utilisé et des nombreuses hypothèses avancées, il est néanmoins possible de dire que l'utilisation de l'effet de la concentration de la solution nutritive apparait surtout comme un moyen de modifier le régime hydrique de la plante ainsi que la morphologie racinaire globale et donc d'obtenir des niveaux photosynthétiques et des degrés de maturité différents chez la vigne.

Une telle considération trouve un potentiel d'application en premier lieu dans les cultures soumises à une irrigation localisée fertilisante où des solutions nutritives trop concentrées devraient en général être évitées, mais aussi dans les cultures non irriguées où une fertilisation localisée en fonction de la nature du sol et du système cultural peut conduire à une exploitation privilégiée de certaines zones de terrain, eu égard au régime hydrique souhaité.

Enfin sur le plan scientifique, cette approche écophysiologique montre la diversité des hypothèses explicatives et la nécessité, en tout cas, de hiérarchiser les questions au niveau de la plante entière avant toute réduction du problème à un seul mécanisme physiologique plausible mais pas obligatoirement déterminant, en l'occurrence ici celui de la réduction des nitrates.

Reçu le 29 iuin 1983. Accepté le 27 février 1984.
Alleweldt G., 1971. Einfluß der N P K Düngung auf die Photosynthese der Reben. Diplomlandwirt Lothar Sautter aus Göppingen, $82 \mathrm{p}$.

Carbonneau A., 1976a. Principes et méthodes de mesure de la surface foliaire. Essai de caractérisation des types de feuilles dans le genre Vitis. Ann. Amélior. Plantes, 26 (2), 327-343.

Carbonneau A., 1976b. Analyse de la croissance des feuilles du sarment de Vigne : estimation de sa surface foliaire par échantillonnage. Conn. Vigne et Vin, 10 (2), 141-159.

Carbonneau A., 1980a. Recherche sur les systèmes de conduite de la Vigne: essai de maitrise du microclimat et de la plante entière pour produire économiquement du raisin de qualité. Thèse Docteur-Ingénieur en Oenologie-Ampélologie, Université de Bordeaux II, $240 \mathrm{p}$.

Carbonneau A., 1980b. Early physiological tests of selection : a key for breeding programs. Proc. 3rd Int. Symp. Grape Breed., Davis (USA), June 15-18 1980. Ed. Univ. Calif., 147-157.

Carbonneau A., 1983. Measurement of gross photosynthesis under natural conditions by using a ${ }^{14} \mathrm{CO}_{2}$ diffusion porometer. I. Description of some convenient techniques. Photosynthetica, 17 (2), 235-239.
Carbonneau A., Casteran P., Leclair P., 1978. Essai de détermination en biologie de la plante entière de relations essentielles entre le bioclimat naturel, la physiologie de la Vigne et la composition du raisin. Méthodologie et premiers résultats sur les systèmes de conduite. Ann. Amélior. Plantes, 28 (2), 195-221.

Carbonneau A., Loth C. de, Smart R. E., 1982. Méthodologie microclimatique utilisable en agrométéorologie ou l'approche de l'écologie quantitative. Vignes Vins, $\mathrm{n}^{\circ}$ spécial de septembre, 87-94

Carbonneau A., Smart R. E., Loth C. de, 1983. Measurement of gross photosynthesis under natural conditions by using a ${ }^{14} \mathrm{CO}_{2}$ diffusion porometer. II. Combined influences of some main ecological factors and leaf area. Photosynthetica, 17 (3), 426-430.

Champagnol F., 1971. Etude de quelques effets de la fertilisation azotée sur la Vigne. Thèse de Docteur-Ingénieur, Université des Sciences et Techniques du Languedoc, $75 \mathrm{p}$.

Champigny M. L., 1960. L'influence de la lumière sur la genèse des acides aminés dans les feuilles de Bryophyllum daigremontianum Berger. Rev. Gen. Bot., 67, 65-215.

Delas J., Pouget R., 1984. Action de la concentration de la solution nutritive sur quelques caractéristiques physiologiques et tech- 
nologiques chez Vitis vinifera $L$. cv. Cabernet-Sauvignon. II. Composition minérale des organes végétatifs, du moût et du vin. Agronomie, 4 (5).

Drew M. C., Saker L. R., 1975. Nutrient supply and the growth of the seminal root system in barley. II. Localized, compensatory increases in lateral root growth and rates of nitrate uptake when nitrate supply is restricted to only part of the root system. $J$. Exp. Bot., 26, 79-90.

Duncan W. G., Ohlrogge A. J., 1958. Principles of nutrient uptake from fertilizer bands. II. Root development in the band. Agron. J., 50, 605-608.
Pouget R., 1984. Action de la concentration de la solution nutritive sur quelques caractéristiques physiologiques et technologiques chez Vitis vinifera $\mathrm{cv}$. Cabernet-Sauvignon. 1. - Vigueur, rendement, qualité du moût et du vin. Agronomie, 4 (5), 437-442.

Richards D., Rowe R. N., 1977a. Effects of root restriction, root pruning and 6-benzylaminopurine on the growth of peach seedlings. Ann. Bot., 41, 729-740.

Richards D., Rowe R. N., 1977b. Root-shoot interactions in peach : the function of the root. Ann. Bot., 41, 1211-1216. 